\title{
Roles of neural precursor cell expressed, developmentally downregulated 9 in tumor-associated cellular processes (Review)
}

\author{
SISEN ZHANG and LIHUA WU \\ Emergency Department, People's Hospital of Zhengzhou, Zhengzhou, Henan 450000, P.R. China
}

Received September 26, 2014; Accepted June 15, 2015

DOI: $10.3892 / \mathrm{mmr} .2015 .4240$

\begin{abstract}
Neural precursor cell expressed, developmentally downregulated 9 (NEDD9), a gene exclusively expressed in the brain during embryonic stages but not in brains of adult mice, is an important cytoskeletal protein and regarded as a 'router/hub' in cellular signal transduction processes connecting external stimulation signals with downstream target proteins that can directly promote tumor metastasis. Numerous studies showed that NEDD9 has an essential role in cell proliferation, apoptosis, adhesion, migration and invasion. The roles of NEDD9, including the underlying mechanisms of its regulation of cell migration, its distinctive functions in various tumor stages and its association with other diseases, are required to be elucidated at large. Future studies of NEDD9 may provide a more profound understanding of the development of tumor invasiveness and NEDD9 may serve as a potential novel target for tumor therapy. The present review examined the significant roles of NEDD9 in the abovementioned processes.
\end{abstract}

\section{Contents}

1. Introduction

2. Regulation of gene expression by NEDD9

3. Roles of NEDD9 in cell migration, adhesion and invasion

4. Roles of NEDD9 in cell apoptosis

5. Roles of NEDD9 in cell cycle control

6. Roles of NEDD9 in development

7. NEDD9 as a target for cancer therapy

Correspondence to: Dr Sisen Zhang, Emergency Department, People's Hospital of Zhengzhou, 33 Yellow River Road, Zhengzhou, Henan 450000, P.R. China

E-mail: sisen1224@sohu.com

Key words: neural precursor cell expressed developmentally downregulated 9, router, cellular signal transduction, stimulation signals, target proteins

\section{Introduction}

Neural precursor cell expressed, developmentally downregulated 9 (NEDD9), a gene exclusively expressed in the brain during embryonic stages but not in the brains of mature mice, was firstly identified in 1992 by Kumar et al (1) by subtractive cloning technology. In 1996, Law et al (2) was the first to ascribe a biological function to NEDD9. They screened a number of genes that can induce the sprouting of filamentous yeast and found a number of proteins that was able to regulate human cell polarity and the cell cycle. Among these proteins, human enhancer of filamentation 1 (HEF1) is expressed in a variety of human cell lines and effectively regulates yeast-cell polarity due to its RecQ C-terminal domain (2). Also in 1996, Minegishi et al (3) identified Crk-associated substrate-related protein lymphocyte type (Cas-L) as they studied a tyrosine hyper-phosphorylated protein under the activation of $\mathrm{T}$ cell $\beta 1$-binding; Cas-L was shown to be identical to NEDD9/HEF1 by sequence alignment (3). Therefore, NEDD9, HEF1 and Cas-L are three different names for the same gene and are used interchangeably.

To date, no evidence has indicated that NEDD, as a cytoskeletal protein, has enzyme activity; however, several structural domains interacting with various proteins have been identified. In vertebrates, the two proteins p130Cas/breast cancer anti-estrogen resistance protein (BCAR)1 (4) and embryonal Fyn-associated substrate (EFS)/Src interacting or signal integrating protein (SIN) (5-7) in NEDD9 and Cas families share quite similar structural domains. p130Cas, as the first identified protein of the Cas family, is expressed in most tissue types and cell lines and contributes to cell adhesion and migration (8). According to sequence analysis, p130Cas and NEDD9 display a high degree of homology. Thus, it was once thought that p130Cas and NEDD9 had similar functions in the regulation of cell adhesion and migration. The present review focused on further in-depth study of NEDD9.

The NEDD9 gene is located in the human chromosome 6p25-24 locus and the overall length of its mRNA is $2505 \mathrm{nt}$, coding a total of 843 amino acids (Fig. 1), where 10-65 amino acids encode the SH3 structural domain (9). At least 15 SH2 structural domains containing 90-350 amino acids, known as the substrate domain (10), interact with proteins containing the SH2 structural domain. The 350-650 amino acids identified by bioinformatics analysis, rich in serine and containing 


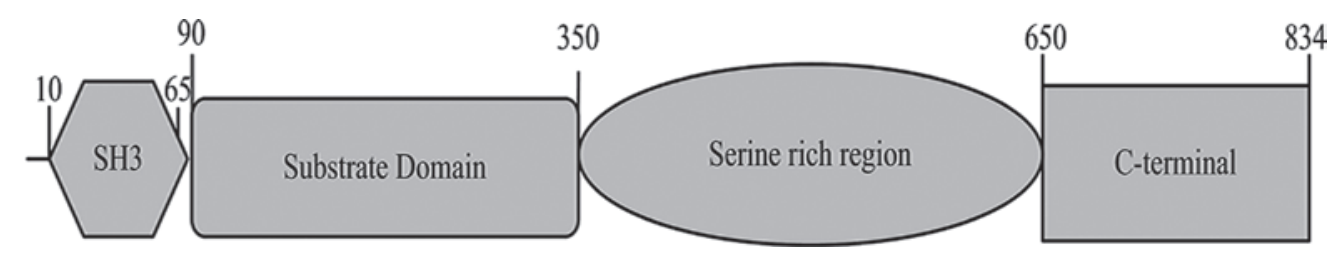

Figure 1. Structure of neural precursor cell expressed developmentally downregulated 9 protein.

four helical structures (11), are likely to be protein binding sites. The c-terminus of NEDD9 is a highly evolutionarily conserved domain that binds with a group of spiral-loop-spiral proteins to form dimers and heterodimers in the Cas protein (12).

\section{Regulation of gene expression by NEDD9}

The regulation of NEDD9 expression is a dynamic complex process, including phosphorylation, transcriptional activation and proteolysis, which exerts a direct or indirect influence on various biological processes. Normally, SDS-PAGE analysis of NEDD9 protein displays two electrophoretic bands at 1,055 and $115 \mathrm{kDa}(13)$, which are above its molecular weight of $93 \mathrm{kDa}$, illustrating that the high degree of phosphorylation has a key role in the regulation of gene expression by NEDD9.

Focal adhesion kinase (FAK) and Src protein families were the first proteins identified to be involved in the regulation of NEDD9 phosphorylation $(14,15)$. In the cell adhesion process, integrin firstly activates FAK; then, the activated FAK generates Src binding sites by tyrosine phosphorylation near the NEDD9 C-terminal domain, and finally, more extensive phosphorylation of the NEDD9 substrate structural domain is generated (Fig. 2).

The phosphorylation of NEDD9 enables it to bind with effector molecules correlated with cell migration, cell invasion and proliferation signaling pathways. In certain types of cancer cell, even without activation by integrin, NEDD9 phosphorylation can be undertaken only through overexpression or activation of FAK and Src (16). As another member of the Cas family, EFS/SIN activates Srs with p130Cas and causes a similar activation effect to that of NEDD9. However, their binding domains with Srs may be different to those of NEDD9 (17). A study showed that FAK acts as an activation agent of NEDD9 (18). NEDD9 phosphorylation is affected by cytoskeleton actin integrity. Actin fracture caused by pharmaceutical substances can result in NEDD9 dephosphorylation. A study by Bargon et al (19) indicated that NEDD9 dephosphorylation brings about a change of Rho kinase activity and a change in the hardness of cytoskeleton actin.

The expression levels of NEDD9 are low in resting cells; however, they sharply increase when cells enter cell cycle (13). Although the regulation of NEDD9 expression has not been thoroughly elucidated, certain inducible factors were found to regulate NEDD9 expression. Transforming growth factor (TGF)- $\beta$ was identified to upregulate NEDD9 mRNA levels and enhance protein expression (20). In two different retinoblastoma cell lines, the metabolite of vitamin A, all-cis retinoic acid (asRA), induced NEDD9 expression, illustrating that NEDD9 expression is associated with nerve-cell development $(21,22)$. In a rat model of cerebral ischemia, NEDD9 was shown to be highly expressed in cerebral cortex and Hippocampal neurons (23).
Studies on ovarian cancer and melanoma cells indicated that enhancement of NEDD9 expression is an important process in promoting cancer metastasis $(18,24)$. (Sex determining region Y)-box 2 and NANOG were also found to combine with the promoter site of NEDD9 (25). However, the functions of these two proteins and their association with cancer are required to be confirmed by further studies.

Negative regulation of NEDD9 levels, including proteolysis or degradation, occurs after gene transcription and results in corresponding decreases in biological function. At the telophase of mitosis, the amount of NEDD9 decreases due to degradation caused by proteolytic enzymes $(13,26)$. During cell apoptosis, the specific DLVD and DDYD cleavage sites for caspase are incised into several short fragments, which negatively regulates the NEDD9 signaling pathway (27).

TGF- $\beta$ signaling pathways also have a role in NEDD9 proteolysis. NEDD9 directly interacts with ubiquitin ligase, Smad proteins and certain factors correlated with target protein degradation or proteolytic cleavage, finally resulting in NEDD9 proteolysis. Furthermore, NEDD9 can regulate the activity of Smad protein as well as inhibit the TGF- $\beta$ signaling pathway (28-32). The close association with TGF- $\beta$ indicates that NEDD9 has an important role in tumor metastasis.

\section{Roles of NEDD9 in cell migration, adhesion and invasion}

Cell migration is a complex process including the change of cell polarity, formation of microfilament and microtubules, and finally more complex regulation, involving cell membrane dynamics and adhesion plaque formation. NEDD9 is located in the cell adhesion plaque and influences cell migration through regulating the interaction of significant molecules that induce cell migration (33). As a normal physiological process of the body, cell migration has a positive impact on embryonic development and the inflammatory response. However, cell migration is abnormally activated in a large proportion of malignant cancer cells, which is attributed to the abnormal regulation of normal cells' non-pathological migration mechanism in metastatic carcinoma. It was also found that changes in NEDD9 expression have an important role in the non-pathological movement of hematopoietic system cells (34), as well as in the migration processes of melanoma (18), breast cancer (35) and glioma (36). The C-terminus of peptides in NEDD9-overexpressing cells can induce the cells to become round and more extended (37), followed by loss of inter-cellular junction adhesion (38). These studies demonstrated that NEDD9, similar to p130Cas (39), directly regulates the formation and dissociation of focal adhesion. In vitro migration and in vivo invasion assays showed that the interaction of NEDD9 and FAK is a crucial initial event during cell migration and invasion processes (40-42). After 


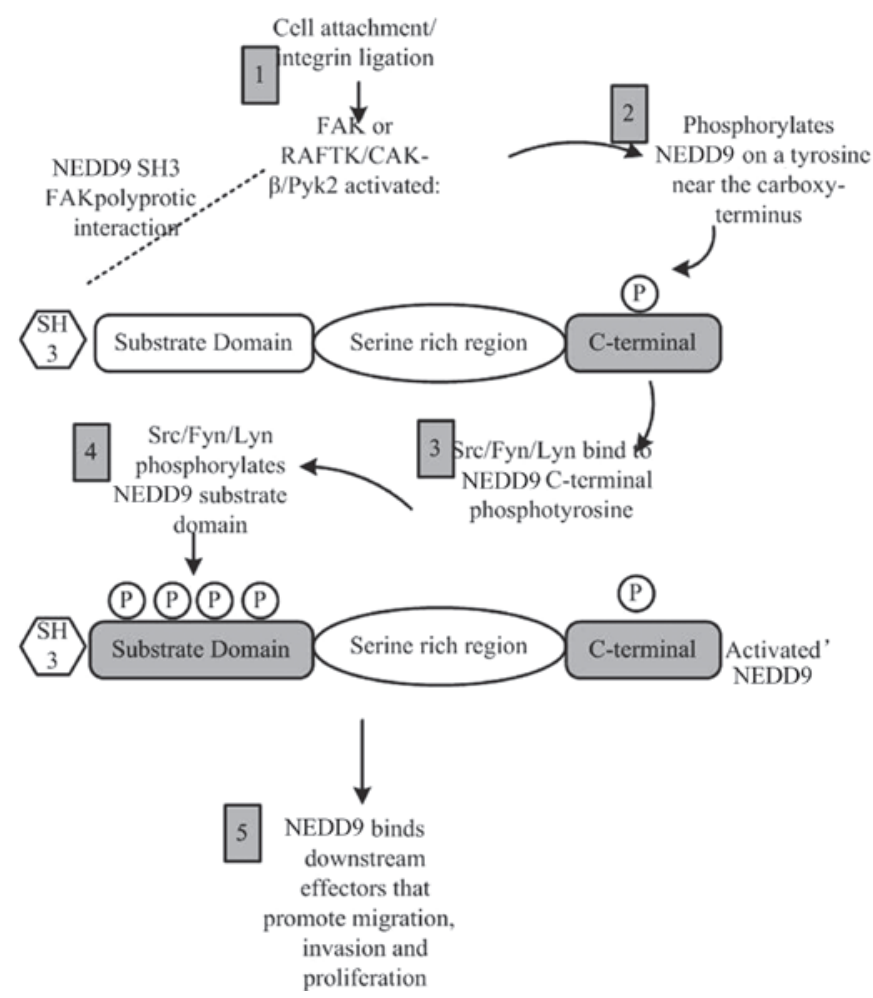

Figure 2. NEDD9 post-translational modification caused by integration NEDD9, neural precursor cell expressed developmentally downregulated 9; FAK, focal adhesion kinase; RAFTK, related adhesion focal tyrosine kinase; $\mathrm{P}$, phosphate; PYK, protein tyrosine kinase; CAK, cell adhesion kinase.

phosphorylation by Src and FAK, NEDD9 directly interacts with adaptor molecule Crk (15). Studies on p130Cas of the Cas family showed that Crk can interact with p130Cas, recruit exchange factor dedicator of cytokinesis 180, activate guanosine triphosphatase (GTPase) Ras-related C3 botulinum toxin substrate (RAC) (43) and finally cause the cell membrane to fluctuate and extend through polymerization of actin-related protein $2 / 3(44,45)$ and activation of p21-activated kinase (46).

In addition, Crk was able to activate $\mathrm{C} 3 \mathrm{G}$ and another migration pathway through GTPase Ras-related protein 1 (Rap1) (47). Even though, the activity of NEDD9 appears similar to that of p130Cas, the mechanisms of the cell migratory pathways stimulated by the two factors require further study. It is noteworthy that the substrate binding site domain of NEDD9 has a binding area that can bind with Crk as well, whereas it remains elusive whether it can cause the activation of Rac and Rap. NEDD9 can also interact with signaling proteins, including BCAR3/AND-34/SHEP2/Nsp2 and CHAT-H/SHEP1, which activate downstream effector molecules through regulating the activity of GTP enzyme (48-51) and finally promote cell migration and invasion.

In vitro experiments showed that NEDD9 overexpression in various cell types can promote cell migration, including the speed of random migration and its tropism (37,40-42), while downregulation of NEDD9 expression can decrease cell chemotaxis (34). It was suggested that the roles of NEDD9 and p130Cas in cell migration are not identical but associated with tissue specificity. For instance, Natarajan et al (36) found that NEDD9 can promote cell migration and invasion in glioma, while p130Cas does not have this function. Another study showed that p130Cas cannot replace the role of NEDD9 in lymphocyte migration in NEDD9 knockout rat models (34).

Inhibition of the expression of Pho kinase, decreased expression of FAK or dominant negative mutation inhibition of FAK can reverse cell migration caused by NEDD9 expression $(18,19,50)$. Of note, in epithelial cells in which Rho expression is inhibited, NEDD9 can induce the formation of neurite-like extensions (19), which indicates that a variety of downstream extension factors may exist. The overexpression of NEDD9 can also activate downstream factors, including mitogen-activated protein kinase, extracellular signal-regulated kinase $1 / 2$ and INK, though the specific function of these factors in the cell migration pathway regulated by NEDD9 has not been elucidated (37). The overexpression of NEDD9 can also activate certain genes with roles in cell migration, including matrix metalloproteinases (MMPs), myosin lightchain kinase, depolymerization-associated genes, Rho kinase, Nck-interacting kinase, receptors of TGF and ErbB2/Her2/Neu receptors (37). Although the precise effects of these cell factors have not been fully elucidated, it was shown that factors including MMPs and depolymerization-associated proteins promote cell migration and invasion (52).

\section{Roles of NEDD9 in cell apoptosis}

Normal cell apoptosis is initiated by caspase cleavage, which is thereby activated and cleaves other proteins and cell components. Apoptosis is accompanied by morphological alterations, including round cell shape, cell membrane protuberances and adhesion plaque decomposition. Cleavage of cell components executed by caspases can cause target molecule activation or inactivation during cell apoptosis. NEDD9 and p130Cas are target proteins for caspases-mediated cleavage $(53,54)$. Cleavage of NEDD9 can inhibit integrin activity, which means that NEDD9 serves as a sensor in the formation of adhesion plaque. Accordingly, inhibition of integrin can cause the activation of caspases (55). In MCF-7 breast cancer cells and other cancer cell types (27), overexpression of the NEDD9 c-terminal $28 \mathrm{kD}$ peptide can induce cell apoptosis. Furthermore, overexpression of whole NEDD9 protein can induce apoptosis as well. It has been suggested that low-level cleavage of NEDD9 generates a small amount of p28 that causes adhesion plaque decomposition and cell apoptosis (56). In MCF-7 cells, NEDD9 initially promotes cell migration and finally causes apoptosis, indicating that the role of NEDD9 is cell cycle-dependent (57). However, studies on Jurkat cells, glioma cells and melanoma cells showed that overexpression of NEDD9 does not result in apoptosis; in contrast to MCF-7 breast cancer cells, which have a relatively low tumor formation ability, these cells are all highly metastatic and invasive (58). A possible explanation for this observation is that cell survival pathway activation is indispensable to the conversion process of the metastatic phenotype in cancer cells. NEDD9 can only promote cancer cell metastasis on the premise of previous survival-pathway activation.

\section{Roles of NEDD9 in cell cycle control}

In the quiescent stage and G1 stage of the cell cycle, the expression of NEDD9 is low. It gradually increases in S stage and reaches a peak in G2/M stage (13). Pugacheva and 


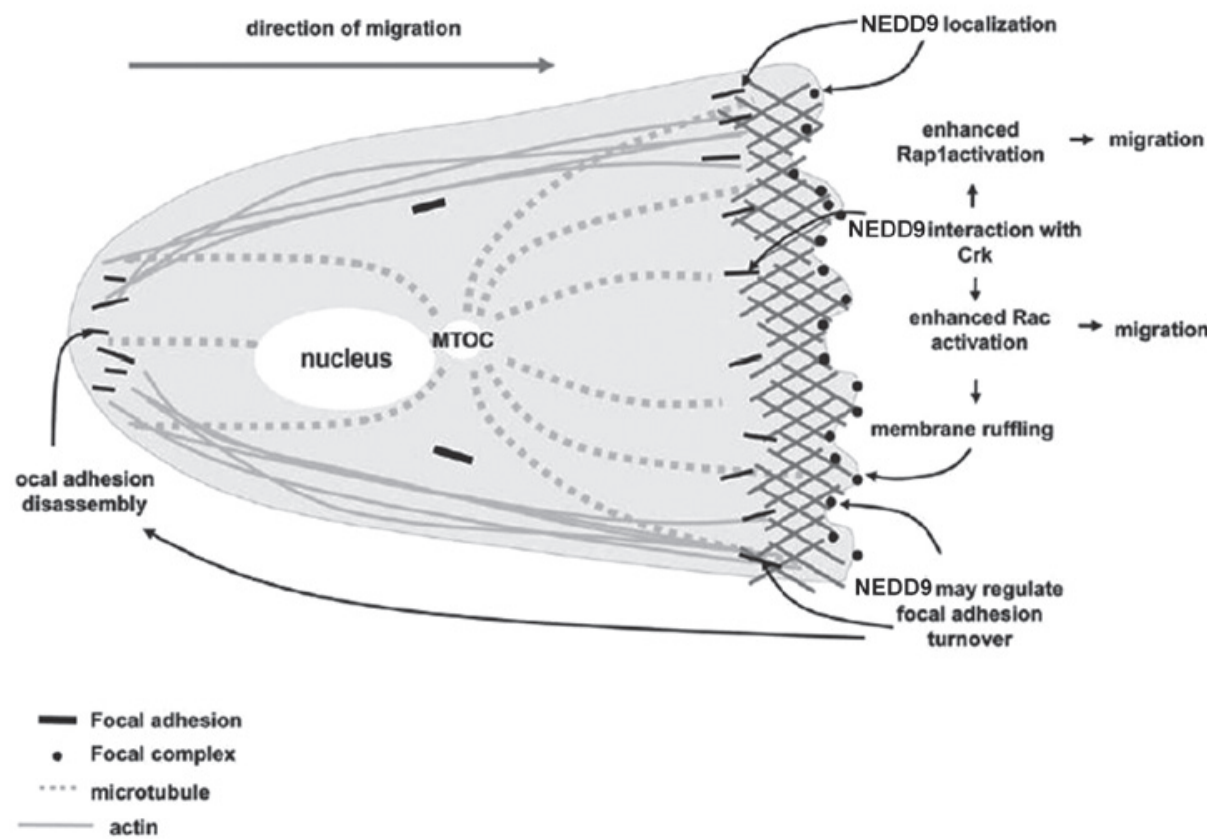

Figure 3. Role of NEDD9 in cell migration. NEDD9, neural precursor cell expressed developmentally downregulated 9; MTOC, microtubule organizing center.

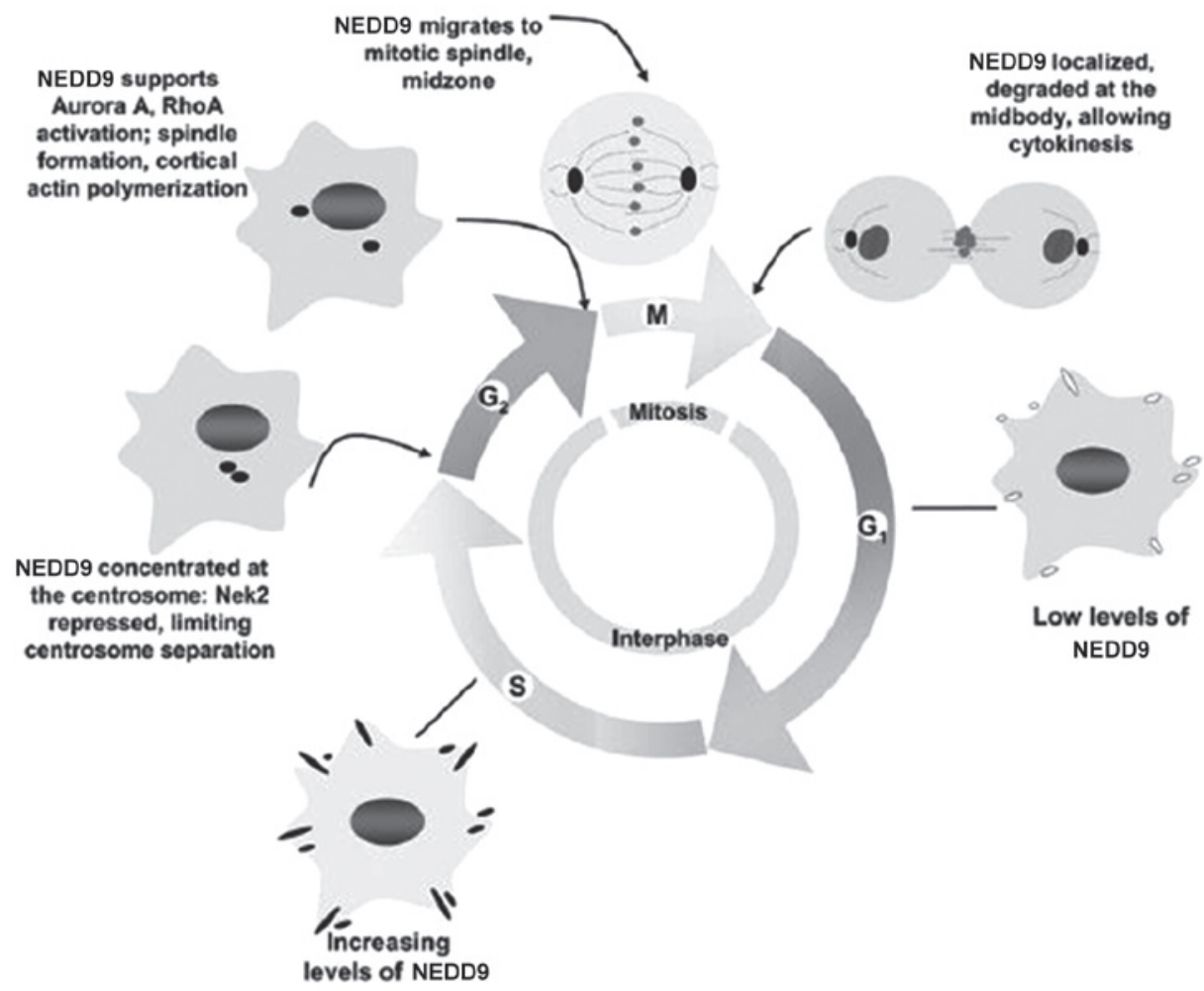

Figure 4. Role of NEDD9 in the cell cycle. NEDD9, neural precursor cell expressed developmentally downregulated 9. RhoA, Ras homolog gene family, member A; Nek2, NIMA-related kinase 2.

Golemis (59) found that NEDD9 mainly lies in the centrosome of mitosis in G2 stage; when mitosis commences, NEDD9 moves to the intermediate zone of mitosis along the spindle; when the cytoplasm divides, NEDD9 is present in the midbody. Overexpression of NEDD9 can increase the number of spindles and centrosomes in mitosis phase, which leads to the failure of cytoplasmic separation (60). However, low expression of NEDD9 can cause pre-mature centrosome separation and a lack of tubulin activation during the separation process, which generates single-stage or asymmetric spindles and leads to cell division failure. Cells with abnormal expression of NEDD9 remain in G1 stage, which is consistent with 
the view that NEDD9 triggers cells to enter mitosis, and due to this cell cycle arrest, cells finally enter apoptosis.

To date, although the precise mechanism of the regulation of the cell cycle by NEDD9 has not been fully elucidated, certain mechanisms have been preliminarily confirmed (Fig. 4). Initially, prior to cell mitosis, NEDD9 combines with centrosome kinase Nek2 to induce Nek2 activation, which results in centrosome separation. In NEDD9-negative cells, NeK2 is prematurely activated and causes the premature separation of the centrosome. Furthermore, during the progression from G2 to $\mathrm{M}$ stage, NEDD9 activates aurora A and the timely activation of aurora A has a crucial role in the process of mitosis. If aurora A cannot be activated in time for cell division, cells present with the same phenotype as that of NEDD9-negative cells (60). Finally, NEDD9 can interact with epithelial cell transforming 2, which can specifically activate RhoA during mitosis (61). The activation of RhoA can regulate several key steps of mitosis. Therefore, overexpression of NEDD9 causes abnormal increases of RhoA activity and cells stay in mitosis phase.

\section{Roles of NEDD9 in development}

NEDD9 also has an important role in signal transduction of developmental cells and non-cancerous cells. Using gene knockout technology, Seo et al (34) found that NEDD9-deficient mice gained enhanced survival and fertility without any obvious tissue abnormalities, while p130Cas-knockout mice died on the eleventh day of the embryonic period (62), which means that the function of NEDD9 can be completely replaced by 130 or other proteins. A great number of studies showed that the deficiency of NEDD9 leads to disturbances in development. Studies on the normal differentiation of nerve cells and brain development showed that NEDD9 has a significant role during this process. Merrill et al $(21,22)$ found that NEDD9 has an important role in brain development by screening all-trans retinoic acid (atRA) in a cDNA-subtractive library, which represents the gene sequence that is expressed in target cells, but not expressed in second cells (different types or cell under different conditions). In the brain developmental process, atRA, as an important regulatory factor, can promote the extension and development of neurites. Upregulation of NEDD9 expression may be a crucial approach to active atRA. Furthermore, NEDD9 also can interact with molecule interacting with Casl, which can regulate the activity of plexin A and activate the semaphorin 3A signaling pathway to regulate the development of the nervous system. Studies of gonadal differentiation in mice also found that NEDD9 is a gender-specific gene; however, the roles of NEDD9 in sexual development require further investigation.

\section{NEDD9 as a target for cancer therapy}

As a cytoskeletal protein, NEDD9 serves as a link in signal transduction processes. NEDD9 contributes to the cell cycle and expression or activation of numerous regulatory proteins. Due to the 'router' function in cells, NEDD9 has a wide-ranging influence on cell proliferation. In the early phase of normal cells and cancer cells, the increased expression of NEDD9 can enhance the migration and invasion abilities of cells.
Furthermore, NEDD9 can initiate post-mitotic defects associated with the failure of cytoplasm movement. Once NEDD9 is fragmented into segments, it causes cell adhesion and apoptosis. NEDD9-overexpressing malignant tumors commonly feature a wide range of pre-cancerous lesions, including the inhibition of p16Ink4, activation of Ras, translation of human T-lymphotropic virus 1 and the BCR-ABL generated by translocation $(18,42,63)$. Hence, cancer cell invasion, apoptosis and cell division can be inhibited through modification of these signaling pathways, which provides novel approaches for inhibiting NEDD9-overexpressing metastatic tumors.

NEDD9 has various roles in tumorigenesis depending on the tumor type; this should be taken into account by clinicians in the interpretation of NEDD9 overexpression in various tumor cell types. For example, in solid tumors, overexpression of NEDD9 may have different biological functions from those in hematopoietic tissue tumors. It is hard to tell whether NEDD9 overexpression is the key factor for tumor invasion in hemocytes, since it is normal for hemocytes to invade other tissues. However, in epithelial cells, NEDD9 can be identified as a biomarker of invasive solid tumors (64).

Previous studies of NEDD9 focused on the first step of cell invasion: Tumor cells escaping from the settlement site $(65,66)$. Therefore, it is likely that activation of NEDD9 may lead to cell migration. The overexpression of NEDD9, a significant biomarker in metastatic melanoma, can promote lung metastases in malignant tumors; however, the processes NEDD9 is involved in are more complex. For example, NEDD9 expression was decreased in the highly metastatic breast cancer cell line MDA-MB231, further indicating that NEDD9 has different roles depending on the tumor type (57).

NEDD9 is a tumor metastasis-promoting gene; however, p130Cas does not have the same function. In normal cells, the expression levels of NEDD9 are maintained in a dynamic balance through transcriptional and proteolytic enzyme degradation regulation (13). In normal cells, various biological effects, including changes of the cell cycle, apoptosis and cell migration, can be achieved by strict regulation of NEDD9 expression.

To date, a profound understanding of the role of NEDD9 in the change of benign to invasive and malignant tumors has been acquired. At the same time, NEDD9 provides a novel target for cancer therapy, particularly that of invasive tumors. However, since NEDD9 does not have any obvious catalytic activity, it remains difficult to target NEDD9 with drugs. However, by blocking the interaction of NEDD9 with other proteins through drugs, or by using RNA interference technology to reduce NEDD9 expression levels may inhibit tumor metastasis.

NEDD9 is probably not an essential protein, as NEDD9 knockout mice can survive, which implies that treating tumors with drugs or through inhibiting NEDD9 expression is feasible. The overexpression of NEDD9 leads to the activation of Ras; therefore, inhibition of the BRC-ABL signaling pathway or Ras function by drugs may produce therapeutic effects against invasive and malignant tumors caused by NEDD9-overexpression $(67,68)$.

There remains a large amount of unanswered questions regarding NEDD9, including the pathways via which it regulates cell migration, its distinctive functions in different tumor stages and its association with other diseases. Further study 
of NEDD9 may provide a more profound understanding of the development of invasive tumors. NEDD9 may serve as a potential novel target for tumor therapy, therefore having a positive significance.

\section{Acknowledgements}

The present review was supported by the Key Technology Research Project of Henan province (grant no. 132102310391).

\section{References}

1. Kumar S, Tomooka Y and Noda M: Identification of a set of genes with developmentally down-regulated expression in the mouse brain. Biochem Biophys Res Commun 185: 1155-1161, 1992.

2. Law SF, Estojak J, Wang B, Mysliwiec T, Kruh G and Golemis EA Human enhancer of filamentation a novel p130cas-like docking protein, associates with focal adhesion kinase and induces pseudohyphal growth in Saccharomyces cerevisiae. Mol Cell Biol 16: $3327-3337,1996$

3. Minegishi M, Tachibana K, Sato T, Iwata S, Nojima Y and Morimoto C: Structure and function of Cas-L, a $105-\mathrm{kD}$ Crk-associated substrate-related protein that is involved in beta-1 integrin-mediated signaling in lymphocytes. J Exp Med 184: $1365-1375,1996$.

4. Kim M, Gans JD, Nogueira C, Wang A, Paik JH, Feng B, Brennan C, Hahn WC, Cordon-Cardo C, Wagner SN, et al: Comparative oncogenomics identifies NEDD9 as a melanoma metastasis gene. Cell 125: 1269-1281, 2006.

5. Sakai R, Iwamatsu A, Hirano N, Ogawa S, Tanaka T, Mano H, Yazaki Y and Hirai $\mathrm{H}$ : A novel signaling molecule, p130, forms stable complexes in vivo with $\mathrm{v}-\mathrm{Crk}$ and $\mathrm{v}-\mathrm{Src}$ in a tyrosine phosphorylation dependent manner. EMBO J 13: 3748-3756, 1994.

6. Alexandropoulos K and Baltimore D: Coordinate activation of c-Src by SH3- and SH2-binding sites on anovel, p130Cas-related protein, Sin. Genes Dev 10: 1341-1341, 1995.

7. Alexandropoulos K, Donlin LT, Xing L and Regelmann AG: Sin: Good or bad? A T lymphocyte perspective. Immunol Rev 192 181-195, 2003

8. Ishino M, Ohba T, Sasaki H and Sasaki T: Molecular cloning of a cDNA encoding a phosphoprotein, Efs, which contains a Src homology 3 domain and associates with Fyn. Oncogene 11: 2331-2338, 1995.

9. Abassi YA, Rehn M, Ekman N, Alitalo K and Vuori K: p130Cas Couples the tyrosine kinase Bmx/Etk with regulation of the actin cytoskeleton and cell migration. J Biol Chem 278: 35636-35643, 2003.

10. Li SS: Specificity and versatility of SH3 and other proline-recognition domains: Structural basis and implications for cellular signal transduction. Biochem J 390: 641-653, 2005.

11. Machida K and Mayer BJ: The SH2 domain: Versatile signaling module and pharmaceutical target. Biochim Biophys Acta 1747: 1-25, 2005.

12. Canutescu AA and Dunbrack RL Jr: MollDE: A homology modeling framework you can click with. Bioinformatics 21 : 2914-2916, 2005

13. Law SF, Zhang YZ, Fashena S, Toby G, Estojak J and Golemis EA: Dimerization of the docking/adaptor protein HEF1/NEDD9/CAS-L via a carboxy-terminal helix-loop-helix domain. Exp Cell Res 252: 224-235, 1999.

14. Law SF, Zhang YZ, Klein-Szanto AJ and Golemis EA: Cell-cycle regulated processing of HEF1 to multiple protein forms differentially targeted to multiple subcellular compartments. Mol Cell Biol 18: 3540-3551, 1998

15. Law SF, Estojak J, Wang B, Mysliwiec T, Kruh G and Golemis EA: Human Enhancer of Filamentation 1 a novel p130cas-like docking protein, associates withfocal adhesion kinase and induces pseudohyphal growth in Saccharomyces cerevisiae. Mol Cell Biol 16: 3327-3337, 1996.

16. Sima N, Cheng X, Ye F, Ma D, Xie X and Lü W: The overexpression of scaffolding protein NEDD9 promotes migration and invasion in cervical cancer via tyrosine phosphorylated FAK and SRC.PLoS One 8: e74594, 2013.

17. Ruest PJ, Shin NY, Polte TR, Zhang $X$ and Hanks SK: Mechanisms of CAS substrate domain tyrosine phosphorylation by FAK and Src. Mol Cell Biol 21: 7641-7652, 2001.
18. Kim M, Gans JD, Nogueira C, Wang A, Paik JH, Feng B Brennan C, Hahn WC, Cordon-Cardo C, Wagner SN, et al: Comparative oncogenomics identifies NEDD9 as a melanoma metastasis gene. Cell 125: 1269-1281, 2006.

19. Bargon SD, Gunning PW and O'Neill GM: The Cas family docking protein, HEF1, promotes the formation of neurite-like membrane extensions. Biochim Biophys Acta 1746: 143-154, 2005.

20. Zheng $M$ and McKeown-Longo PJ: Regulation of HEF1/NEDD9/CAS-L expression and phosphorylation by TGF-beta 1 and cell adhesion. J Biol Chem 277: 39599-39608, 2002.

21. Merrill RA, See AW, Wertheim ML and Clagett-Dame M: Crk-associated substrate (Cas) family member, NEDD9, is regulated in human neuroblastoma cells and in the embryonic hindbrain by all-trans retinoic acid. Dev Dyn 231: 564-575, 2004.

22. Merrill RA, Ahrens JM, Kaiser ME, Federhart KS, Poon VY and Clagett-Dame M: All-trans retinoic acid-responsive genes identified in the human SH-SY5Y neuroblastoma cell line and their regulated expression in the nervous system of early embryos. Biol Chem 385: 605-614, 2004.

23. Sasaki T, Iwata S, Okano HJ, Urasaki Y, Hamada J, Tanaka H, Dang NH, Okano H and Morimoto C: Nedd9 protein, a Cas-L homologue, is upregulated after transient global ischemia in rats. Possible involvement of Nedd9 in the differentiation of neurons after ischemia. Stroke 36: 2457-2462, 2005

24. Donninger H, Bonome T, Radonovich M, Pise-Masison CA, Brady J, Shih JH, Barrett JC and Birrer MJ: Whole genome expression profiling of advance stage papillary serous ovarian cancer reveals activated pathways. Oncogene 23: 8065-8077, 2004.

25. Boyer LA, Lee TI, Cole MF, Johnstone SE, Levine SS, Zucker JP, Guenther MG, Kumar RM, Murray HL, Jenner RG, et al: Core transcriptional regulatory circuitry in human embryonic stem cells. Cell 122: 947-956, 2005.

26. Pugacheva EN and Golemis EA: The focal adhesion scaffolding protein HEF1 regulates activation of the Aurora-A and Nek2 kinases at the centrosome. Nat Cell Biol 7: 937-946, 2005.

27. Law SF, O'Neill GM, Fashena SJ, Einarson MB and Golemis EA: The docking protein HEF1 is an apoptotic mediator at focal adhesion sites. Mol Cell Biol 20: 5184-5195, 2000.

28. Nourry C, Maksumova L, Pang M, Liu X and Wang T: Direct interaction between Smad3, APC10, CDH1 and HEF1 in proteasomal Degradation of HEF1. BMC Cell Biol 5: 20, 2004.

29. Liu X, Elia AE, Law SF, Golemis EA, Farley J and Wang T: A novel ability of $S m a d 3$ to regulate proteasomal degradation of a cas family member, HEF1. EMBO J 19: 6759-6769, 2000.

30. Feng L, Guedes S and Wang T: Atrophin-1-interacting protein 4/human Itch is a ubiquitin E3 ligase for human enhancer of filamentation 1 in transforming growth factor-beta signaling pathways. J Biol Chem 279: 29681-29690, 2009.

31. Inamoto $S$, Iwata $S$, Inamoto $T$, Nomura $S$, Sasaki $T$, Urasaki Y, Hosono O, Kawasaki H, Tanaka H, Dang NH, et al: Crk-associated substrate lymphocyte type regulates transforming growth factor-beta signaling by inhibiting Smad6 and Smad7. Oncogene 26: 893-904, 2006.

32. Wang T: The 26S proteasome system in the signaling pathways of TGF-beta superfamily. Front Biosci 8: 1109-1127, 2003.

33. Wozniak MA, Modzelewska K, Kwong L and Keely PJ: Focal adhesion regulation of cell behavior. Biochim Biophys Acta 1692: 103-119, 2004.

34. Seo S, Asai T, Saito T, Suzuki T, Morishita Y, Nakamoto T, Ichikawa M, Yamamoto G, Kawazu M, Yamagata T, et al: Crk-associated substrate lymphocyte type is required for lymphocyte trafficking and marginal zone B cell maintenance. J Immunol 175: 3492-3501, 2005.

35. Minn AJ, Gupta GP, Siegel PM, Bos PD, Shu W, Giri DD, Viale A, Olshen AB, Gerald WL and Massagué J: Genes that mediate breast cancer metastasis to lung. Nature 436: 518-524, 2005.

36. Natarajan M, Stewart JE, Golemis EA, Pugacheva EN, Alexandropoulos K, Cox BD, Wang W, Grammer JR and Gladson CL: HEF1 is a necessary and specific downstream effector of FAK that promotes the migration of glioblastoma cells. Oncogene 25: 1721-1732, 2006.

37. Fashena SJ, Einarson MB, O'Neill GM, Patriotis C and Golemis EA: Dissection of HEF1-dependent functions in motility and transcriptional regulation. J Cell Sci 115: 99-111, 2002.

38. O'Neill GM and Golemis EA: Proteolysis of the docking protein HEF1 and implications for focal adhesion dynamics. Mol Cell Biol 21: 5094-5108, 2001. 
39. Webb DJ, Donais K, Whitmore LA, Thomas SM, Turner CE, Parsons JT and Horwitz AF: FAK-Src signalling through paxillin, ERK and MLCK regulates adhesion disassembly. Nat Cell Biol 6: 154-161, 2004

40. Van Seventer GA, Salman HJ, Law SF, et al: Focal adhesion kinase regulates betal integrin dependent migration through an HEF1/NEDD9/CAS-L effector pathway. Eur J Imm 31: 1417-1427, 2001

41. Ohashi Y, Iwata S, Kamiguchi K and Morimoto C: Tyrosine phosphorylation of Crk-associated substrate lymphocyte-type is a critical element in TCR- and betal integrin-induced T lymphocyte migration. J Immunol 163: 3727-3734, 1999.

42. Iwata S, Souta-Kuribara A, Yamakawa A, Sasaki T, Shimizu T, Hosono O, Kawasaki H, Tanaka H, Dang NH, Watanabe T, et al: HTLV-I Tax induces and associates with Crk-associated substrate lymphocyte type (Cas-L). Oncogene 24: 1262-1271, 2005.

43. Klemke RL, Leng J, Molander R, Brooks PC, Vuori K and Cheresh DA: CAS/Crk coupling serves as a 'molecular switch' for induction of cell migration. J Cell Biol 140: 961-972, 1998.

44. Ridley AJ: Rho proteins: Linking signaling with membrane trafficking. Traffic 2: 303-310, 2001.

45. Smith LG and Li R: Actin polymerization: Riding the wave. Curr Biol 14: R109-R111, 2004.

46. Cai D, Iyer A, Felekkis KN, Near RI, Luo Z, Chernoff J, Albanese C, Pestell RG and Lerner A: AND-34/BCAR3, a GDP exchange factor whose overexpression confers anti estrogen resistance, activates Rac, PAK1 and the cyclin D1 promoter. Cancer Res 63: 6802-6808, 2003.

47. Tamada M, Sheetz MP and Sawada Y: Activation of a signaling cascade by cytoskeleton stretch. Dev Cell 7: 709-718, 2004.

48. Cai D, Felekkis KN, Near RI, O'Neill GM, van Seventer JM, Golemis EA and Lerner A: The GDP exchange factor AND-34 is expressed in B cells, associates with HEF1 and activates Cdc42. J Immunol 170: 969-978, 2003.

49. Gotoh T, Cai D, Tian X, Feig LA and Lerner A: p130Cas regulates the activity of AND-34, a novel Ral, Rap1 and R-Ras guanine nucleotide exchange factor. J Biol Chem 275: 30118-30123, 2000

50. Sakakibara A, Hattori S, Nakamura S and Katagiri T: A novel hematopoietic adaptor protein, Chat-H, positively regulates $\mathrm{T}$ cell receptor-mediated interleukin-2 production by Jurkat cells. J Biol Chem 278: 6012-6017, 2003.

51. Sakakibara A and Hattori S: Chat, a Cas/HEF1-associated adaptor protein that integrates multiple signaling pathways. J Biol Chem 275: 6404-6410, 2000.

52. Lucas JT Jr, Salimath BP, Slomiany MG and Rosenzweig SA: Regulation of invasive behavior by vascular endothelial growth factor is HEF1-dependent. Oncogene 29: 4449-4459, 2010.

53. Gervais FG, Thornberry NA, Ruffolo SC, Nicholson DW and Roy S: Caspases cleave focal adhesion kinase during apoptosis to generate a FRNK-like polypeptide. J Biol Chem 273 17102-17108, 1998.
54. Kook S, Shim SR, Choi SJ, Ahnn J, Kim JI, Eom SH, Jung YK, Paik SG and Song WK: Caspase-mediated cleavage of p130Cas in etoposide-induced apoptotic Rat-1 cells. Mol Biol Cell 11: 929-939, 2000

55. Stupack DG, Puente XS, Boutsaboualoy S, Storgard CM and Cheresh DA: Apoptosis of adherent cells by recruitment of caspase-8 to unligated integrins. J Cell Biol 155: 459-470, 2001.

56. Frisch SM: Anoikis. Methods Enzymol 322: 472-429, 2000.

57. Chang JX, Gao F, Zhao GQ and Zhang GJ: Role of NEDD9 in invasion and metastasis of lung adenocarcinoma. Exp Ther Med 4: 795-800, 2012

58. Biscardi JS, Belsches AP and Parsons SJ: Characterization of human epidermal growth factor receptor and c-Src interactions in human breast tumor cells. Mol Carcinog 21: 261-272, 1998.

59. Pugacheva EN and Golemis EA: HEF1-aurora A interactions: Points of dialog between the cell cycle and cell attachment signaling networks. Cell Cycle 5: 384-391, 2006.

60. Dadke D, Jarnik M, Pugacheva EN, Singh MK and Golemis EA: Deregulation of HEF1 impairs M-phase progression by disrupting the RhoA activation cycle. Mol Biol Cell 17: 1204-1217, 2006.

61. Fritz G and Kaina B: Rho GTPases: Promising cellular targets for novel anticancer drugs. Curr Cancer Drug Targets 6: 1-14, 2006.

62. Honda H, Oda H, Nakamoto T, Honda Z, Sakai R, Suzuki T, Saito T, Nakamura K, Nakao K, Ishikawa T, et al: Cardiovascular anomaly, impaired actin bundling and resistance to Src-induced transformation in mice lacking p130Cas. Nat Genet 19: 361-365, 1998.

63. Dail M, Kalo MS, Seddon JA, Côté JF, Vuori K and Pasquale EB: SHEP1 function in cell migration is impaired by a single amino acid mutation that disrupts association with the scaffolding protein cas but not with Ras GTPases. J Biol Chem 279: 41892-41902, 2004.

64. Speranza MC, Frattini V, Pisati F, Kapetis D, Porrati P, Eoli M, Pellegatta S and Finocchiaro G: NEDD9, a novel target of miR-145, increases the invasiveness of glioblastoma. Oncotarget 3: 723-734, 2012.

65. Chang JX, Gao F, Zhao GQ and Zhang GJ: Expression and clinical significance of NEDD9 in lung tissues. Med Oncol 29: 2654-2660, 2012

66. Liu Y, Wang D, Zhao KL, Zhu JW, Yin HB, Wei YZ, Wu ZJ, Cheng GJ, Wang F, Ni F, et al: NEDD9 overexpression correlates with poor prognosis in gastric cancer. Tumour Biol 35: 6351-6356, 2014.

67. Kato-Stankiewicz J, Hakimi I, Zhi G, Zhang J, Serebriiskii I, Guo L, Edamatsu H, Koide H, Menon S, Eckl R, et al: Inhibitors of Ras/Raf-1 interaction identified by two-hybrid screening revert Ras-dependent transformation phenotypes in human cancer cells. Proc Natl Acad Sci USA 99: 14398-14403, 2002.

68. Druker BJ: Perspectives on the development of a molecularly targeted agent. Cancer Cell 1: 31-36, 2002. 